\title{
EL CAMPO DE SABER ECONÓMICO. UNA APROXIMACIÓN DESDE LA EPISTEMOLOGÍA
}

\author{
CÉSAR CARRANZA \\ Flacso-Ecuador
}

\begin{abstract}
Recepción manuscrito: 1 de julio de 2014
\end{abstract}
Aceptación versión final: 30 de octubre de 2014

\begin{abstract}
RESUMEN El artículo gira en torno a los elementos ontológicos y epistemológicos que dieron surgimiento al «campo de saber de la economía», proceso que ha estado cargado de un marcado positivismo y que incidió en una separación de la Economía de las otras ciencias sociales. Este distanciamiento impuso una forma única de entender los procesos sociales y económicos, reduciendo los comportamientos, las prácticas y las relaciones económicas a una visión axiomática. Por otro lado, distintas voces provenientes de la academia y de otros sectores han ido demandando la presencia de enfoques plurales y críticos dentro de la Economía.
\end{abstract}

PALABRAS ClAVE Economía crítica, heterodoxia, academia, epistemología.

ABSTRACT This paper focuses on the ontological and epistemological elements that gave rise to the «field of knowledge economy» process that has been loaded with a marked positivism and that influenced separation of economics from other social sciences. This distancing imposed a unique way of understanding the social and economic processes, reducing behaviors, practices and economic relations to an axiomatic vision. Furthermore, different voices from academia and other sectors have been demanding the presence of plural and critical approaches within Economics.

KEY WORDS Critical economy, heterodoxy, academy, epistemology.

JEL CODE B5O, A22, A23

\section{INTRODUCCIÓN}

Más de uno, como yo sin duda, escriben para perder el rostro. No me pregunten quién soy, ni me pidan que permanezca invariable. [...] Que nos deje en paz cuando se trata de escribir.

M. Foucault

La tendencia en la formación y en los círculos académicos de Economía está dominada por la perspectiva ortodoxa, caracterizada principalmente por el análisis marginalista y la escuela 
neoclásica, la cual es vista y promovida como la única aproximación válida y científica para analizar los hechos económicos, el predominio de esta perspectiva va acompañada de la deslegitimación de cualquier otro abordaje teórico y epistemológico y las prácticas que no se ajustan a sus supuestos son vistas como anacrónicas, anormales, irracionales o reminiscencias de un pasado a superar. La vigencia del enfoque económico convencional está también sostenida por su relación con una perspectiva política que busca legitimar su posición e imponer una manera específica de entender el mundo, los problemas sociales, en este caso los procesos y acontecimientos económicos. No debemos olvidar que cualquier campo científico está mediatizado por la lucha por la legitimidad, y estos procesos de legitimidad se dan en confrontaciones políticas, de las cuales lo científico no está ajeno; atrás de las diferentes posturas académicas hay luchas políticas, a las cuales la ciencia sucumbe. La idea positivista de una ciencia neutral y acumulativa no es real.

La importancia de contar con perspectivas epistemológicas alternativas y críticas en Economía y en las ciencias sociales, y que éstas se vayan estructurando como un corpus sistemático de pensamiento ha ido creciendo en la actualidad, no solo por las limitaciones del enfoque principal, sino por su poca capacidad para dar respuesta a necesidades diversas en un mundo heterogéneo, con inequidades, problemas sociales, ambientales y económicos que ponen entredicho los modelos tradicionales de desarrollo y los supuestos y recomendaciones de política promovidos desde el enfoque convencional.

REFLEXIONES EN TORNO AL SURGIMIENTO DE LA ECONOMÍA CONVENCIONAL

El marcado positivismo que caracterizó la formulación y desarrollo de la teoría económica neoclásica, se tradujo en un progresivo distanciamiento de la Economía y del entendimiento de lo económico del resto de disciplinas y ciencias sociales. Esta perspectiva conceptual, denominada también como utilitarista, formal o mainstream, plantea una dicotomía permanente entre utilidad y escasez, donde los individuos aparecen enfrentados a la naturaleza y compitiendo entre ellos por bienes escasos; el utilitarismo se sustenta en una visión axiomática que reduce y simplifica el comportamiento de todos los individuos y de los procesos asociados a la toma de decisiones, las cuales se las realiza (de acuerdo a este enfoque) de manera individual, movidas por la maximización de beneficios y de utilidad.

Esta perspectiva postula la existencia de una racionalidad universal caracterizada por el Homo œconomicus, al cual se lo describe como un ser egoísta que se preocupa únicamente por sus propios beneficios y que tiene preferencias (sobre bienes y servicios) completas, continuas y fijadas exógenamente. Este ser, que continuamente está maximizando, no se preocupa del bienestar social a menos que afecte su bienestar o consumo individual.

Privada del contenido de las relaciones sociales, incapaz de incorporar su historia y dar cuenta de ella, la definición «formal» de la Economía se enviste de toda la vieja mitología del Homo œconomicus que expresa y legitima la visión «burguesa» de la sociedad y de la «racionalidad» económica, entendida como maximización del beneficio de los individuos o de los grupos sociales que se enfrentan en la competencia en el seno de una sociedad reducida a un mercado (de bienes, de poder, de valores, etc.) (Godelier, 1976, p. 284). 


\section{ELEMENTOS ONTOLÓGICOS Y EPISTEMOLÓGICOS DEL ENFOQUE ORTODOXO}

\section{[...] Un [auténtico] revolucionario en materia científica es alguien que tiene} un gran dominio de la tradición ( $y$ no alguien que hace tabla rasa del pasado o que, más simplemente, lo ignora).

P. Bourdieu

Existe un consenso en varios autores (Munti y Barrionuevo, 2010; Teira Serrano, 2009) en considerar que las bases metodológicas y epistemológicas del mainstream de la Economía se encuentran en los trabajos de Milton Friedman y Paul Samuelson. En particular, la obra de Friedman, Ensayos de Economía Positiva (1953), es considerada como la obra de mayor repercusión e influencia en el siglo XX en metodología económica (Teira Serrano, 2009). En este texto, Friedman, desde una perspectiva positivista e instrumental, plantea que las teorías deberían ser juzgadas por su coherencia lógica y capacidad predictiva y no por la realidad o consecuencias de sus supuestos (lo que se conoce como instrumentalismo), y que la economía positiva (la cual se sustenta en las formalizaciones y modelizaciones matemáticas) tiene la capacidad de brindar a los investigadores los elementos y bases para desarrollar predicciones certeras; de esta manera la Economía podría constituirse en una ciencia objetiva equiparable a las ciencias naturales.

Por su parte, Samuelson, con su propuesta y formalización de la preferencia revelada, buscó dotar de contenido empírico a la teoría del comportamiento del consumidor, a través del análisis de sus hábitos de compra; su trabajo Fundamentos del Análisis Económico (1947) realiza lo que se ha denominado la síntesis neoclásica (articulación de la microeconomía walrasiana y la macroeconomía keynesiana), para lo cual empleó instrumentos matemáticos. Para este autor la matematización de la Economía era el camino para llegar a consecuencias contrastables empíricamente. Posteriormente, Samuelson publica su trabajo Problemas de Metodología (1963) en el cual refuta el planteamiento instrumental de Friedman, planteando que cuando lo contrastamos con la realidad es la teoría y no los supuestos los que están siendo sometidos a prueba.

Tanto Friedman como Samuelson contribuyen con sus aportes a dar un sustento metodológico a la economía neoclásica, más allá de las diferencias y controversia existentes entre sus propuestas, los dos comparten un marco ontológico que plantea la modelización matemática como base interpretativa, la cual ineludiblemente es sostenida como parte del «núcleo duro» de la corriente principal (Munti y Barrionuevo, 2010, p. 2).

Dentro de las reflexiones y debates metodológicos y epistemológicos de los economistas del mainstream durante buena parte del siglo XX, está presente la influencia de Popper, Kuhn y Lakatos, cuyos planteamientos repercutieron en el trabajo de varios economistas renombrados; por ejemplo, Popper (su propuesta de epistemología del racionalismo crítico), en Friedman y principalmente en Hayeck (con quien mantuvo una estrecha amistad); Kuhn (con su noción de paradigma, ciencia normal y revolución científica) en Hicks, Simon y Joan Robinson; Lakatos con su planteamiento de Metodología de Programas de Investigación Científica ${ }^{1}$ (González, 2009).

Esta construcción intelectual remite a una concepción epistemológica que alude a un orden armónico simbolizado por la mano invisible del mercado. La asignación de recursos tiene lugar por la realidad «natural» del intercambio de mercancías. Tal enfoque invoca la realización de 
la libertad del mercado. La base conceptual de la teoría neoclásica se halla, por una parte, en la impersonalidad y objetividad de la racionalidad formal, y por otra, en una concepción mecanicista de la economía que explica el protagonismo del concepto de equilibrio competitivo (Brunet y Pastor, 2001, p. 166)

\section{ENFOQUES HETERODOXOS EN ECONOMÍA}

Desde un sector de la academia y desde otros sectores sociales e intelectuales, progresivamente se ha ido demandando la incorporación y recuperación de enfoques plurales, alternativos y críticos de la Economía que le permitan tener una mayor correspondencia con el «mundo real»y donde esté integrada al sistema social; esta perspectiva (o conjunto de enfoques alternativos) se los han definido como heterodoxos.

Lo heterodoxo es un «concepto» que se define de manera negativa (en oposición a lo ortodoxo) y que a la vez es fragmentado ya que incorpora a diversas tradiciones de pensamiento. De esta manera, enfoques tan diversos como el sustantivista, el poskeynesianismo, el marxismo, el estructuralismo latinoamericano, la economía ecológica, la economía social y solidaria, la economía feminista, la economía institucional evolutiva e incluso la escuela austriaca, entre otros, son considerados o se autodefinen como heterodoxos. Las diferencias y límites entre un enfoque u otro, incluyendo los métodos e instrumentos utilizados, pueden en algunos casos ser claros, pero no siempre, incluso en relación a la perspectiva ortodoxa, por lo que algunos trabajos heterodoxos podrían estar más cercanos a los abordajes del mainstream. Asimismo, las recomendaciones de política y de acción propuestas por las diferentes tradiciones heterodoxas para un mismo tema pueden ser diversas e incluso contrapuestas entre sí.

Dentro de la conformación de este corpus teórico heterodoxo, podemos identificar una intersección entre algunos de los desarrollos presentados por el estructuralismo latinoamericano con los aportes realizados a la macroeconomía por la llamada corriente poskeynesiana, la cual toma como referencia metodológica la idea de construir modelos que expliquen los fenómenos económicos sobre supuestos de mayor apego a la realidad, al caracterizar al capitalismo por la naturaleza imperfecta de los mercados, por el papel determinante de la distribución del ingreso sobre la formación de los precios y el ritmo de la actividad económica, y por la importancia de la incertidumbre que obliga a buscar mecanismos institucionales de defensa. Estos aspectos constitutivos son comunes con la macroeconomía estructuralista, desde la cual se han construido explicaciones para destacar ciertas especificidades de los problemas macroeconómicos de los países de la periferia.

Existen autores contemporáneos - de la Escuela de Cambridge, como Lawson $(2003,2005)^{2}$ que proponen que la diferencia entre la ortodoxia y heterodoxia en Economía es fundamentalmente ontológica; siendo necesaria desde esta perspectiva la necesidad de un "giro ontológico» que conlleve posteriormente la adopción de una posición epistemológica (el autor propone el realismo crítico) y metodológica (Modelo Transformacional de la Actividad Social) alternativas.

Hasta ahora, las diferentes escuelas heterodoxas -el institucionalismo, austrianismo, poskeynesianismo, evolucionismo, feminismo, entre otros- aparecían como reacciones aisladas a la tradición neoclásica, siendo muchas veces cuestionadas o marginadas por no ofrecer críticas «sistemáticas» a la economía tradicional, e incluso por las supuestas contradicciones en 
que han incurrido algunas veces sus propios seguidores. A través de su argumento, Lawson le otorga una coherencia y cohesión a la crítica heterodoxa que hasta hace muy poco no poseía. En efecto, su noción de una economía heterodoxa unida por una base ontológica común, ha contribuido a dar una gran fuerza y presencia a los enfoques alternativos al mainstream, que en los últimos años han experimentado una renovada y saludable expansión (Perona, 2005, p. 15).

Asimismo, autores provenientes de la New School for Social Research, como Duncan Foley (1991, 2010), han trabajado elementos conceptuales y metodológicos de la economía heterodoxa, principalmente desde una aproximación neomarxiana; planteando una ruptura con la noción del homo ceconomicus y proponiendo interpretaciones multicausales y alternativas a los periodos de crisis del capitalismo (Foley, 2010).

Dentro de la comunidad heterodoxa existen diversas voces, algunas orientadas a buscar generar un enfoque unificado en base a un conjunto de principios compartidos y aceptados por todos, mientras que otros ven en esta heterogeneidad y en la contraposición al enfoque dominante los elementos que definen y potencian esta perspectiva. Para alguno autores (Lawson, 2003, 2005), por ejemplo, es la ruptura ontológica que se puede identificar en las diversas tradiciones heterodoxas lo que puede integrarlas como un corpus de pensamiento.

La importancia de contar con perspectivas alternativas en Economía y que éstas se vayan estructurando como un corpus sistemático de pensamiento ha ido creciendo en la actualidad, no solo por las limitaciones del enfoque ortodoxo, sino por su poca capacidad para dar respuesta a necesidades diversas en un mundo heterogéneo, con inequidades, problemas sociales, ambientales y económicos que ponen entredicho los modelos tradicionales de desarrollo.

Durante la última década la comunidad de economía heterodoxa ha crecido, diversificado e integrado. Quienes anteriormente estaban aislados, ahora forman parte de una comunidad; las asociaciones heterodoxas existen en países donde antes no existían, y los desarrollos en la teoría heterodoxa y en política están ocurriendo a una velocidad vertiginosa. En resumen, la economía heterodoxa es ahora una característica establecida en el panorama de la disciplina y progresivamente el futuro de la ciencia económica. (Lee y Jo, 2013, p. 7; traducción del autor).

\section{ACADEMIA Y MOVIMIENTO POSTAUTISTA}

Los cuestionamientos al mainstream en Economía y a la homogenización de este enfoque en la academia han ido ganando fuerza durante los últimos años. Así por ejemplo, en mayo del 2000, estudiantes de economía de universidades francesas, respaldados por varios profesores, promovieron el autodenominado movimiento de «economía postautista», en cuyo manifiesto planteaban la necesidad de superar el estado de ensimismamiento y de alejamiento de la «realidad económica» en que se encontraba la Economía y su enseñanza como disciplina en los círculos académicos ${ }^{3}$. Los cuatro aspectos que se cuestionaron respecto con la enseñanza de Economía fueron los siguientes:

- El distanciamiento y visión fragmentada de la perspectiva enseñada en clases respecto de la realidad económica y social

- El excesivo uso de instrumentos matemáticos, los mismos que en los procesos de enseñanza 
son entendidos como fines en sí mismos, con los cuales se construyen modelos formales ajenos y estilizados de la realidad con poca capacidad explicativa de procesos complejos.

- El dogmatismo en la enseñanza, sin espacios críticos.

- La conformidad y letargo de los docentes.

Este movimiento se trasladó rápidamente a otros lugares; así, en Cambridge durante el 2001 un manifiesto similar fue promovido por estudiantes y profesores del doctorado de Economía, en el 2003 en España (el cual tomó como nombre Movimiento para una Economía Crítica), ese mismo año en Estados Unidos (Universidad de Kansas y con un círculo de estudiantes de Harvard). En la actualidad prácticamente es un movimiento a nivel global. El movimiento de Cambridge generó una red global en la web que actualmente se denomina de «Economía Real», estableció una revista académica indexada (Real-World Economics Review) y desde el 2011 se conformó la Asociación Mundial de Economía (World Economics Association) con un enfoque plural, crítico y heterodoxo.

En Latinoamérica, movimientos similares surgieron principalmente en Buenos Aires y Ciudad de México, los cuales, por ejemplo, han permitido la realización de varios encuentros y jornadas de economía crítica y han promovido una discusión inicial para la modificación del currículo en las facultades de Economía en varias universidades de distintos países. Así mismo se han establecido varias redes, blogs y asociaciones de economía heterodoxa. En Brasil, destaca la Universidad de Campinas-UNICAMP de Sao Paulo, la que ha incorporado dentro de su currículo de Economía especialmente en posgrado y doctorado un enfoque totalmente heterodoxo .

En Argentina, desde el 2007 se vienen desarrollando de manera anual Jornadas de Economía Crítica (JEC), con el objetivo de generar un espacio pluralista de discusión de los hechos económicos, en un inicio estas Jornadas fueron promovidas por las Escuelas de Economía Política de la Universidad de Buenos Aires (UBA) y de la Universidad de La Plata (UNLP); en el 2013 se constituye la Sociedad de Economía Crítica de la Argentina, incorporando a las principales universidades del país. Las VII-JEC se desarrollan en la ciudad de La Plata a mediados de octubre de 2014. Así mismo, desde el 2010 se viene desarrollando un debate en torno a los contenidos del plan de estudios en las facultades de Economía de las principales universidades del país buscando la incorporación de enfoques alternativos y críticos a más de los planteamientos e instrumental metodológico del enfoque del mainstream.

[...] Existe una tendencia hacia un modelo de pensamiento único, que se asume como sentido común, lo que se ve agravado por conducirnos a la reproducción de lo ya existente. En todas las universidades argentinas, los planes de estudios tienden a imponer los contenidos epistémicos de la escuela neoclásica (síntesis neoclásica, nueva economía clásica). Así se conforma una ortodoxia que no cuestiona ciertos fundamentos (utilitarismo, marginalismo, individualismo metodológico, racionalidad instrumental, entre otros) y que no admite variantes. Esto transforma el plan de economía en la aplicación repetida de un mismo patrón a infinidad de cuestiones (la maximización sujeta a restricciones), partiendo y sosteniendo en todo momento la misma matriz de análisis; sin cuestionar sus fundamentos ni hacer explicita esta elección —dando lugar a la idea de que se trata de una presentación objetiva de «lo que la 
economía es»- - . Por último, y no trivial, estas corrientes proponen un enfoque donde las clases sociales no son consideradas como relevantes, y menos aún el conflicto social en forma de enfrentamiento de clases. Los contenidos heterodoxos suelen estar confinados a materias optativas o eventualmente a una comisión de una materia. No quedan plasmados de modo sistemático a lo largo del plan de estudios (JEC, 2010, p. 2).

\section{REFLEXIONES FINALES}

La invitación debe hacerse y debe aceptarse.

M. Mauss

Las actividades económicas no pueden desligarse de su contexto social, quizá éste, al igual que la pretensión de universalización de sus supuestos, son los mayores sesgos del corpus teórico de la economía neoclásica. A nivel público, las preferencias económicas no dominan, puesto que dependen del contexto, de igual manera, las actitudes emotivas, que frecuentemente dominan las decisiones, son contexto-dependientes.

El predominio del enfoque dominante crea la convención social en la profesión de que sólo la producción de cocimiento económico que se ajusta a dicho enfoque puede ser buena investigación, por lo que todas las demás formas de conocimiento económico simplemente se rechazan por considerarse pobres o algo ajeno a la Economía. Muchos economistas se tienen por tanto que enfrentar al dilema de usar lo que consideran métodos inapropiados de enfrentarse a las cuestiones económicas o adoptar los métodos que ellos consideran mejores para el problema en cuestión sabiendo que su trabajo probablemente no será escuchado por los economistas (Carta Abierta de los Estudiantes de Cambridge; citada en Guerrero, 2002, p. 18).

Las palabras, los conceptos y enunciados de la economía y de lo económico no están aislados, están contenidos y contienen los acontecimientos que designan, estos conceptos explican aquello que ayudan a crear; en este sentido el lenguaje tiene un "poder» puesto que es el vehículo que impone sentidos para entender la realidad y estructurar comprensiones, en nuestro caso de lo económico. Los conceptos emergen de la confrontación de fuerzas sociales y políticas por capturar el sentido de una situación. Los campos de saber, que incluyen a los sujetos de saber y sus de las prácticas que racionaliza, convergen en la producción de un sentido de verdad, la que opera como una mecanismo de legitimación, naturalización, y propician modos de institucionalización. De esta manera, el sujeto de saber participa activamente, conscientemente, en el establecimiento de un mundo, al que considera por sí como verdadero.

Un campo de saber no solo se decide sobre que percibir, o comprender, sino sobre los modos de intervención, de operación de ese saber, en el terreno de las prácticas; esto es, se propone una manera de actuar sobre una realidad específica por medio de la generación.

Foucault (2012 [2004]), identifica al conocimiento y poder como elementos indisolublemente unidos, de manera que uno no tiene sentido sin el otro, donde los dos están al mismo nivel, sin tener uno supremacía o soberanía sobre el otro. El conocimiento, el saber y la ciencia se convierten de esta forma en elementos primados para materializar formas más efectivas del ejercicio del poder y por tanto de la política. El mercado como el nuevo dispositivo de control. 
Siguiendo a Rancière, uno debe volverse a preguntar cosas que ya parecen resueltas, superadas y dadas, en un ejercicio que se asemeja a una genealogía histórica, un recorrido que hace que el pensamiento se piense, interrogándose el cómo se muestra en la actualidad ese pensamiento, cómo se produce ese saber, un ejercicio de refundar los objetos de pensamiento, en nuestro caso el pensamiento económico, dentro del cual existen axiomas clásicos y fundantes que frecuentemente son considerados como verdades inamovibles y que son aceptados y reproducidos dentro del ejercicio académico de manera automática, en un actuar que se asemeja más a un acto de fe, que a un ejercicio de producción intelectual de pensamiento.

Pese a los distintos cuestionamientos y evidencias que deslegitiman la universalidad de los planteamientos y axiomas que sustentan el corpus teórico de la economía neoclásica, ésta es la visión dominante, considerada como única, válida y científica para entender los procesos económicos, incluso en contextos sociales y culturales diversos. Esta lógica del mainstream en la cual todo puede ser valorado, cuantificado, tranzado y modelado, aún sistemas complejos y abiertos como el económico y social, donde priman valores múltiples, con sus retroalimentaciones, interdependencias y no linealidades, se mantiene paradójicamente incluso en momentos en que su capacidad de acción y predicción han sido cuestionados por la crisis económica y financiera internacional. Es por esto, que podemos metaforizar la capacidad de resiliencia, de regeneración y de absorción de la visión neoclásica de la Economía y de sus supuestos (donde tautológicamente podemos introducir cualquier cosa en la noción de utilidad y en la que en sus modelizaciones «todo es posible» dependiendo de los parámetros utilizados) con la mítica hidra de Lerna, en la cual de cada cabeza cortada, de cada supuesto desmoronado, surgen dos.

Todos estos elementos nos llevan a considerar que los planteamientos de la economía neoclásica son insuficientes para entender otras formas de economía y a lo económico desde una perspectiva amplia integrada al sistema social. Este ejercicio demanda de una ruptura paradigmática y epistemológica, en la cual no basta con suavizar o relativizar sus supuestos fundantes, de lo contrario «es algo así como reconstruir una casa, desde los cimientos hasta el techo, mientras se está viviendo en ella todavía» (Polanyi, 1976, p. 288).

La ruptura epistémica dentro de la Economía, no puede realizarse desconociendo o simplemente deslegitimando los supuestos, los axiomas, la racionalidad y el corpus teórico de la economía tradicional. Este es un ejercicio de libertad, «solo si nos apropiamos de los instrumentos de pensamiento, y también de los objetos de pensamiento que recibimos, podremos convertirnos un poco en el sujeto de nuestros pensamientos» (Bourdieu y Chartier, 2011, p. 38).

El trabajo que proponemos es, por tanto, una historia critica del saber económico. Dilucidar su emergencia, sus lógicas, sus objetos y sus objetivaciones, las redes institucionales en las que se riegan y racionalizan, los sujetos que la hacen posible. Se trata de analizar los juegos de relaciones entre saberes y poderes, entre instituciones y sujetos de saber específicos. Es decir, los modos de operación que los regímenes de saber diagraman en lo social, institucional y político. Se trata de objetivar las relaciones entre el saber y los poderes, la manera en como las 'síntesis conceptuales' buscan imponer sentidos de verdad y como se traducen en operaciones concretas como políticas económicas, políticas sociales, reordenamiento institucional, etc. 


\section{NOTAS}

1 Que corresponde de acuerdo a la perspectiva de Lakatos, a un conjunto de teorías interrelacionadas que tienen tanto un «núcleo duro» de supuestos provisionalmente aceptados que se consideran como irrefutables y un «cinturón protector» de hipótesis auxiliares para hacer frente a las anomalías.

2 Lawson tiene formación inicial en matemáticas y luego en economía; plantea que los métodos formales y matematizados que emplea el mainstream son inapropiados para trabajar con la Economía ya que forma parte de un sistema abierto (el social) y que no son aplicables siempre y en cualquier lugar; así mismo cuestiona el individualismo metodológico de la ortodoxia, los axiomas de racionalidad y la tenencia a buscar equilibrios. Su obra es considera como de las de mayor repercusión y trascendencia actual en la heterodoxia económica (Munt y Barrionuevo, 2010; Perona, 2005)

3 El Manifiesto del Movimiento Postautista en Economía dice lo sugiente: «Nosotros, estudiantes de Economía en las universidades y grandes escuelas francesas, nos declaramos globalmente descontentos de la enseñanza que recibimos en ellas. Y ello por las siguientes razones: 1) ¡Salgamos de los mundos imaginarios! [...], 2) ¡No al uso incontrolado de las matemáticas! [...], 3) ¡Por un enfoque plural en Economía! Demasiado a menudo, la lección magistral no deja espacio a la reflexión. Entre todos los enfoques que existen, sólo se nos presenta uno, que se supone capaz de explicar todo según un método puramente axiomático, como si se tratase de LA verdad económica. Nosotros no aceptamos ese dogmatismo. Queremos un pluralismo en las explicaciones, adaptado a la complejidad de los objetos y a la incertidumbre que planea sobre la mayoría de las cuestiones en economía; 4) Llamada a los docentes: ¡Despiértense, antes de que sea demasiado tarde! [...] No queremos seguir fingiendo que estudiamos esta ciencia autista que tratan de imponernos. No pedimos lo imposible, sino tan sólo lo que el buen sentido nos sugiere a todos. Esperamos por tanto que se nos escuche lo antes posible».

\section{BIBLIOGRAFÍA}

Bourdieu, P. y Chartier, R. (2011). El sociólogo y el historiador. Madrid, España: Abada Editores.

Bourdieu, P. (2003). El oficio del científico. Ciencia de la ciencia y reflexividad. Barcelona, España: Anagrama.

Brunet, I. y Pastor, I. (2001). La Axiomática de la Ciencia Económica Convencional. Política y Sociedad, (37), 161-179.

Foley, D. (2010). «The political economy of post-crisis global capitalism», presentado en la conferencia de Economy and Society en la University of Chicago, 3-5 de diciembre. http://homepage.newschool.edu/ foleyd/FoleyPolEconGlobalCap.pdf

Foley, D. (1991). Notes on Ideology and Methodology. Department of Economics, New School for Social Research, New York, 1991. https://docs.google.com/a/newschool.edu/viewer?a=v\&pid=sites\&sr cid=bmV3c2Nob29sLmVk dXxkdW5jYW4tZm9sZXkta.

Godelier, M. (1976). Antropología y Economía. ¿Es posible la Antropología Económica? En Godelier, M. (Comp.) Antropología y Economía. Barcelona, España: Editorial Anagrama.

González, W. (2009): La Repercusión de Popper, Kuhn y Lakatos en la metodología de la Economía. En C. García Bermejo (Ed.). Sobre la economía y sus métodos (p. 327 -354). Madrid, España: Editorial Trotta.

Guerrero, D. (2002). «Autismo, Matemáticas y Microeconomía. Primeras reflexiones sobre un nuevo movimiento de economía crítica», Ponencia presentada en VIII Jornadas de Economía Crítica, enero de 2002, Valladolid. 
JEC (2010). Por un cambio en la formación de Economía (Argentina). Recuperado de: http://esepuba. files.wordpress. com/2010/05/documento-planes-de-estudio-mdp-0103.pdf

Kuhn, T. (2004). La estructura de las revoluciones científicas. Buenos Aires, Argentina: Fondo de Cultura Económica.

Munt, J. y Barrionuevo, G. (noviembre, 2010): Reflexiones sobre la ontología de la Economía: La visión tradicional vs. La heterodoxia moderna. KAIROS Revista de Temas Sociales, 14(26).

Perona, E. (2005). El debate en torno a la propuesta de Tony Lawson para Reorientar la Economía. Empresa y Humanismo, 9(2-05).

Rancière, J. (1993). Los nombres de la historia. Una poética del saber. Buenos Aires, Argentina: Nueva Visión.

Tae-Hee, Jo (Ed.) (2013). Heterodox Economics Directory, 5th Edition, January 2013, http://heterodoxnews.com/ direc-tory/hed5.pdf

Teira Serrano, D. (2009). Los economistas como metodólogos. C. García Bermejo (Ed.). Sobre la economía y sus métodos (p. 21-34). Madrid, España: Editorial Trotta. 